\title{
BMJ
}

\section{Early waning of maternal measles antibodies in era of measles elimination: longitudinal study}

\author{
E Leuridan, pre-doctoral fellow, ${ }^{1} \mathrm{~N}$ Hens, professor in biostatistics and evidence-based vaccinology, ${ }^{2} \mathrm{~V}$ Hutse, \\ pre-doctoral fellow, ${ }^{3} \mathrm{M}$ leven, professor in medicine, ${ }^{4} \mathrm{M}$ Aerts, professor in biostatistics, ${ }^{5} \mathrm{P}$ Van Damme, \\ professor in medicine/vaccinology ${ }^{1}$
}

${ }^{1}$ Centre for the Evaluation of Vaccination, Vaccine and Infectious Disease Institute, Faculty of Medicine, University of Antwerp, Universiteitsplein, 1, 2610 Wilrijk, Belgium

${ }^{2}$ Interuniversity Institute for Biostatistics and Statistical Bioinformatics (I-BIOSTAT),

Hasselt University, Campus Diepenbeek, Centre for Health Economics Research and

Modelling Infectious Diseases,

Vaccine and Infectious Disease Institute, University of Antwerp

${ }^{3}$ National Laboratory for Measles and Rubella, Scientific Institute of

Public Health, Programme of Virology, Brussels, Belgium

${ }^{4}$ Department of Medical Microbiology, Vaccine and Infectious Disease Institute, Faculty of Medicine, University of Antwerp

${ }^{5}$ Interuniversity Institute for Biostatistics and Statistical Bioinformatics (I-BIOSTAT), Hasselt University

Correspondence to: E Leuridan elke.leuridan@ua.ac.be

Cite this as: BMJ 2010;340:c1626 doi:10.1136/bmi.c1626

\section{ABSTRACT}

Objective To investigate the duration of the presence of maternal antibodies to measles in infants.

Design Prospective study (May 2006 to November 2008).

Setting Five hospitals in the Province of Antwerp,

Belgium.

Participants of 221 pregnant women recruited, 207

healthy woman-infant pairs were included-divided into a vaccinated group $(n=87)$ and naturally immune group ( $n=120)$, according to vaccination documents and history. Main outcome measure Measles IgG antibodies measured by enzyme linked immunosorbent assay (ELISA) at seven time points (week 36 of pregnancy, birth (cord), and 1, 6, 9, and 12 months); decay of maternal antibody in infants modelled with linear mixed models. Results Vaccinated women had significantly fewer IgG antibodies (geometric mean titre 779 (95\% confidence interval 581 to 1045$) \mathrm{mIU} / \mathrm{ml}$ ) than did naturally immune women (2687 (2126 to 3373) $\mathrm{mlU} / \mathrm{ml})(\mathrm{P}<0.001)$.

Maternal values were highly correlated with neonatal values ( $r=0.93$ at birth). Infants of vaccinated women had significantly lower antibody concentrations than did infants of naturally immune women ( $\mathrm{P}<0.001$ at all ages over the follow-up period). Presence of maternal antibodies endured for a median of 2.61 months-3. 78 months for infants of naturally infected women and 0 . 97 months for infants of vaccinated women. At 6 months of age, more than $99 \%$ of infants of vaccinated women and $95 \%$ of infants of naturally immune women had lost maternal antibodies according to the model.

Conclusions This study describes a very early susceptibility to measles in infants of both vaccinated women and women with naturally acquired immunity. This finding is important in view of recent outbreaks and is an argument for timeliness of the first dose of a measles vaccine and vaccination of travelling or migrating children under the age of 1 year.

\section{INTRODUCTION}

Reduction of measles related mortality is one of the World Health Organization's goals. Measles vaccination is administered globally with good results: between 2000 and 2007, deaths from measles fell by $74 \%$ worldwide. ${ }^{1}$ However, measles is re-emerging in countries with low incidence and two dose vaccination programmes in place, as shown by recent outbreaks. ${ }^{2}$

Primary protection against infectious diseases at birth is provided mainly by maternal antibodies. ${ }^{3}$ These antibodies could hamper the humoral antibody response of the infant to vaccination, ${ }^{4-6}$ so the timing of vaccination should take their presence into consideration. Several factors determine the amount of maternal antibodies in young infants. Gestational age defines placental transfer: preterm neonates receive significantly fewer antibodies. ${ }^{7}$ The coverage of universal immunisation programmes influences the amount of maternal antibodies: a higher coverage reduces the probability of natural boosting. The mean age at first pregnancy is increasing, and the time since maternal measles vaccination is increasing with it. Finally, the rate of decay of maternal antibodies after birth defines the duration of protection in infants.

International differences in the prevalence of maternal measles antibodies in infants have been described. ${ }^{89}$ Results of previous studies show lower starting concentrations at birth and faster decay of antibodies in infants of vaccinated women. ${ }^{8}$ As many countries implement universal measles immunisation of infants at the age of 12-15 months, the possibly increasing gap of susceptibility due to the early loss of maternal antibodies is of increasing concern. In addition, several studies report a considerable delay in administration of a first dose of vaccine in infants, which is increasing the gap even more. ${ }^{1011}$ In Flanders, Belgium, measles vaccination was recommended at the age of 15 months within the universal vaccination programme after 1984 . Since 2002, this first dose has been administered at 12 months. ${ }^{12}$ A second dose at the age of 11-12 years has been recommended since $1994 .{ }^{13}$

The objective of this study was to investigate the duration of the presence of maternal antibodies to measles in infants on the basis of measurement at seven time points (a longitudinal study). We compared the amount and kinetics of maternal antibodies in the offspring of vaccinated women with those in the offspring of women with naturally acquired immunity. We used a modelling system to predict the time to 
loss of maternal antibodies in infants on the basis of the maternal antibody titre.

\section{METHODS}

Study design

The main aim of this prospective study was to investigate differences in maternal antibodies in infants of vaccinated women and naturally infected women. According to a sample size calculation, we needed at least 200 participants to detect a $10 \%$ difference in geometric mean titre between the two groups of infants with a probability of $99 \%$.

We included healthy pregnant women and their healthy offspring, starting in April 2006 and with follow-up until November 2008. Exclusion criteria were impaired immunology in mother or child, administration of immunoglobulins or blood products during the study period, preterm delivery ( $<36$ weeks), and low birth weight $(<2400 \mathrm{~g})$. Inclusion criteria were age 18-40 years and residence in Belgium for the duration of the study. Participants completed a questionnaire on demographics, validated vaccination history, and medical history. Growth parameters, breast feeding, day care attendance, immunisation data, and medical histories for all household members were registered at each visit.

We classified women into two groups. One group was vaccinated during infancy, and we sought vaccination documents for each woman. The other group had natural immunity to measles. We presumed natural infection taking a history of it in the woman and her parents. We included women of foreign origin only if they had written proof of vaccination or history of disease. All participants and both parents of the participating children gave informed consent.

We collected venous whole blood during pregnancy (week $36,10 \mathrm{ml}$ ), at birth (10 ml of cord blood), and in all infants ( $2 \mathrm{ml}$ ) at months 1 (27-34 days), 3 (84-99 days ), and 12 (358-372 days) and randomly at either month 6 (175-189 days) or 9 (267-282 days). All samples except for cord blood were collected during home visits. Samples were centrifuged at $2000 \mathrm{rpm}$ within eight hours after sampling and stored at $-20^{\circ} \mathrm{C}$.

\section{Determination of antibodies}

We used an enzyme linked immunosorbent assay (ELISA; Enzygnost Anti-Measles IgG, Dade Behring, Germany) for quantitative measurement of measles IgG antibodies. The analysis was done at the Programme of Virology, Scientific Institute of Public Health and the University Hospital of Antwerp. We calibrated the assay against the international reference preparation of measles antigen and present results in milli-international units per millilitre. If corrected optical density was greater than 0.2 , we considered the sample to be positive; if the corrected optical density was less than 0.1 , the sample was negative. We considered samples with optical density between 0.1 and 0.2 to be inconclusive. We used kit dependent parameters to calculate quantitative results. Quantitative results could not be trusted for corrected optical densities below 0.1 . We set the estimated "protective" threshold at $300 \mathrm{mIU} / \mathrm{ml}$ on the basis of the optical density measurements. A protective threshold ELISA value for measles has not been established. The same test is used at the Institute of Public Health in Belgium for surveillance purposes, making comparison with other national data possible.

\section{Statistical analyses}

We did two statistical analyses. The first analysis modelled the decay of maternal antibodies in infants with time, taking into account heterogeneity among infants and homogeneity within infants. We used a linear mixed model to relate the log maternal antibody concentration of the infant over time and other potentially influential factors. We used the Akaike information criterion to select the appropriate model by using a forward selection procedure up to the third order for all factors. This allows for the estimation of individual specific profiles over time. ${ }^{14}$ Missing values are taken into account by assuming that they are missing at random ("direct likelihood" approach). ${ }^{14}$ We used a log transformation of the antibody concentration- $\log (\mathrm{AB}+1)$ - to symmetrise the response distribution. We assessed the validity of the assumption of normality by using the Kolmogorov-Smirnov test $(\mathrm{P}=0.14)$. We considered $\mathrm{P}$ values $<0.01$ to be significant. We used a likelihood ratio test to test for a hospital specific random effect. In the second analysis, on the basis of the antibody decay model from the first analysis and assuming that immunity was lost whenever the antibody concentration fell below $300 \mathrm{mIU} / \mathrm{ml}$, we used normal regression to estimate the individual time to loss of immunity in relation to the log antibody concentration of the mother.

\section{RESULTS}

\section{Population}

We included 221 women and their 226 infants. We excluded from the analysis women who had received vaccination and had natural infection, as well as women who had neither vaccination nor natural infection, with their infants $(n=7)$. We also excluded woman-child pairs of which the infant(s) met exclusion criteria $(n=9)$. We analysed data from 207 women and their 210 infants; 120 women belonged to the naturally immune group, and 87 were vaccinated. All vaccinated women had received a single dose of measles vaccine during childhood. Their mean age at immunisation was 3.3 years (median 1.6 years). We included three foreign women, two originating from the Netherlands and one from the United Kingdom. Samples were missed from $13 \%$ of the infants at different time points owing to difficulty with drawing blood.

Table 1 shows the general characteristics of the women and their infants. Seventy-six per cent (158/ 207) of all participating women were expecting a first child, $16 \%(33 / 207)$ a second child, and $6 \%(12 / 207)$ a third child. 
Table 1|General characteristics of women and infants

\begin{tabular}{|c|c|c|c|c|}
\hline Characteristics & All women $(n=207)$ & $\begin{array}{l}\text { Vaccinated women (VACC) } \\
\qquad(\mathrm{n}=87)\end{array}$ & $\begin{array}{l}\text { Naturally immune women } \\
\text { (NAT) }(n=120)\end{array}$ & $\begin{array}{l}\text { P value for difference } \\
\text { (VACC-NAT) }\end{array}$ \\
\hline $\begin{array}{l}\text { Mean (range) age of women } \\
\text { (years) }\end{array}$ & $30(23-41)$ & $28(23-35)$ & $32(24-41)$ & $0.267^{\star}$ \\
\hline No (\%) primiparous & $161(77)$ & $76(85)$ & $85(70)$ & $0.018^{\star}$ \\
\hline No (\%) caesarean section & $64(20)$ & $23(26)$ & $18(15)$ & $0.104^{*}$ \\
\hline No of infants & 210 & 87 & 123 & \\
\hline Girls/boys & $113 / 97$ & $45 / 42$ & $68 / 55$ & $0.79 *$ \\
\hline Mean gestational age & 39 weeks, 4 days & 39 weeks, 4 days & 39 weeks, 4 days & $0.56 \dagger$ \\
\hline Mean birth weight (g) & 3365 & 3407 & 3335 & $0.33 \dagger$ \\
\hline $\begin{array}{l}\text { Mean duration breast } \\
\text { feeding (weeks) }\end{array}$ & 19.5 & $20.0(n=71)$ & $19.1(n=107)$ & $0.76 \dagger$ \\
\hline No (\%) day care attendance & $158(76)$ & $70(80)$ & $88(73)$ & $0.9^{*}$ \\
\hline
\end{tabular}

\section{Serological results}

The overall geometric mean titre of IgG at week 36 of pregnancy was 1593 (95\% confidence interval 1306 to 1944) $\mathrm{mIU} / \mathrm{ml} ; 8 \%$ of naturally immune women and $26 \%$ of vaccinated women did not reach the threshold in the ELISA test $(\mathrm{IgG} \leq 300 \mathrm{mIU} / \mathrm{ml})$. The geometric mean titre in women differed significantly between vaccinated women (779 (581 to 1045$) \mathrm{mIU} / \mathrm{ml}$ ) and naturally immune women (2687 (2126 to 3373) IU/ ml) $(\mathrm{P}<0.001)$. Maternal concentrations were highly associated with neonatal concentrations at birth $(r=0.93)$ : infants of vaccinated women had significantly fewer antibodies at birth compared with infants of naturally immune women $(\mathrm{P}<0.001)$ (table 2$)$.

At the age of 3 months, 21/73 (29\%) available samples of infants of vaccinated women were still considered positive compared with 51/85 (60\%) samples of infants of naturally immune women. At the age of 6 months, 11/72 (15\%) samples were positive, all but one child from naturally immune mothers. At months 9 and 12, no positive samples remained in either group. The geometric mean titre still differed significantly between the groups of infants with increasing age. However, interpretation should be cautious as the later titres were below $300 \mathrm{mIU} / \mathrm{ml}$ for both groups and not reproducible with the ELISA test.

\section{Model based analysis}

The final linear mixed model included all interactions of time and log antibody concentration of the mother. Breast feeding, parity, gestational age, birth weight, educational level, day care attendance, and caesarean section did not have a significant impact. Although mothers were clustered within hospitals, we found no significant cluster effect $(\mathrm{P}=0.0845)$. Figure 1 depicts the resulting profiles.

The rate of decay of maternal antibodies can be calculated from the fitted model and was on average steeper in infants of naturally immune women than in infants of vaccinated women (derivations not shown). This is implicitly reflected in figure 2 , in which the proportion immune for the infants of naturally immune women starts higher and finally reaches the proportion immune for the infants of vaccinated women after seven months. According to the model, the overall median time to loss of immunity was 2.61 months. On the basis of these predicted profiles, the time to decrease below the threshold of the protective amount of antibodies can be estimated. Figure 2 shows the proportion of infants immune to measles against the time to loss of passive acquired immunity. Median time to immunity loss was 3.78 months for infants of naturally immune women and 0.97 months for infants of vaccinated women. At 6 months of age, more than $99 \%$ in

Table 2 | Geometric mean titre (GMT) of IgG against measles and proportion of positive samples at each time point for all women, naturally infected women, and vaccinated women

\begin{tabular}{|c|c|c|c|c|}
\hline \multirow[b]{2}{*}{ Time point } & \multicolumn{3}{|c|}{ GMT in $\mathrm{mIU} / \mathrm{ml}(95 \% \mathrm{CI})$ (No of positive samples*/total No of tested samples) } & \multirow{2}{*}{$\begin{array}{l}\text { P value } † \text { for difference in GMT } \\
\text { (VACC-NAT) }\end{array}$} \\
\hline & All women & Vaccinated women (VACC) & Naturally immune women (NAT) & \\
\hline Women at week 36 of pregnancy & $1593(1306$ to 1944$)(181 / 214)$ & 779 (581 to 1045) (67/90) & 2687 (2126 to 3373) (114/124) & $<0.001$ \\
\hline Cord & 1369 (1106 to 1695$)(152 / 189)$ & $698(521$ to 935$)(55 / 79)$ & 2221 (1702 to 2899$)(97 / 110)$ & $<0.001$ \\
\hline Month 1 & $928(735$ to 1173$)(122 / 160)$ & $493(364$ to 668$)(41 / 67)$ & $1463(1076$ to 1989$)(81 / 93)$ & $<0.001$ \\
\hline Month 3 & $304(238$ to 387$)(72 / 158)$ & 179 (132 to 243$)(21 / 73)$ & 477 (340 to 670) (51/85) & 0.003 \\
\hline Month 6 & $79(58$ to 108$)(11 / 72)$ & $37(27$ to 50$)(1 / 31)$ & $142(94$ to 215$)(10 / 41)$ & 0.01 \\
\hline Month 9 & $15(12$ to 18$)(0 / 89)$ & $12(8$ to 16$)(0 / 44)$ & 19 (14 to 24$)(0 / 45)$ & 0.03 \\
\hline Month 12 & 11 (6 to 9) (0/156) & $6(4$ to 8$)(0 / 68)$ & $9(7$ to 11$)(0 / 88)$ & 0.04 \\
\hline
\end{tabular}



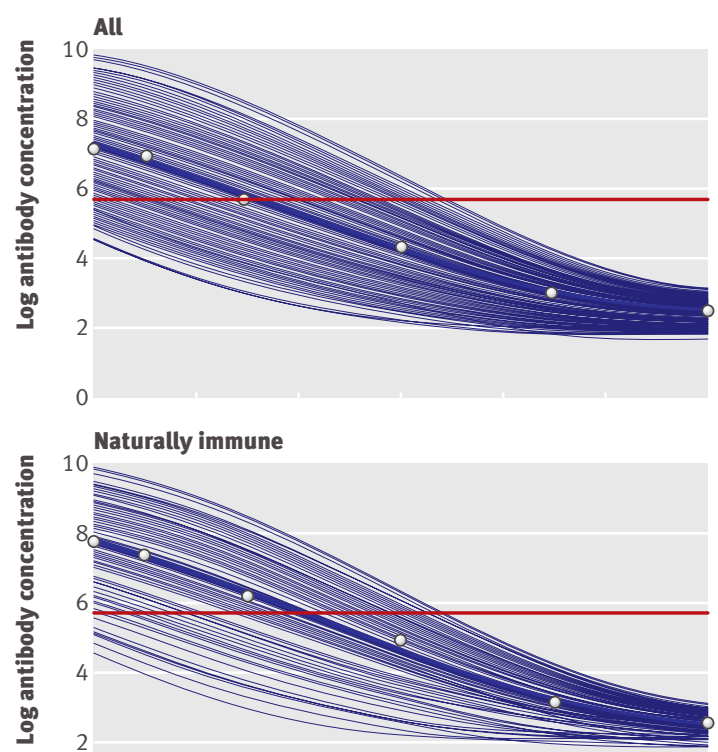

0

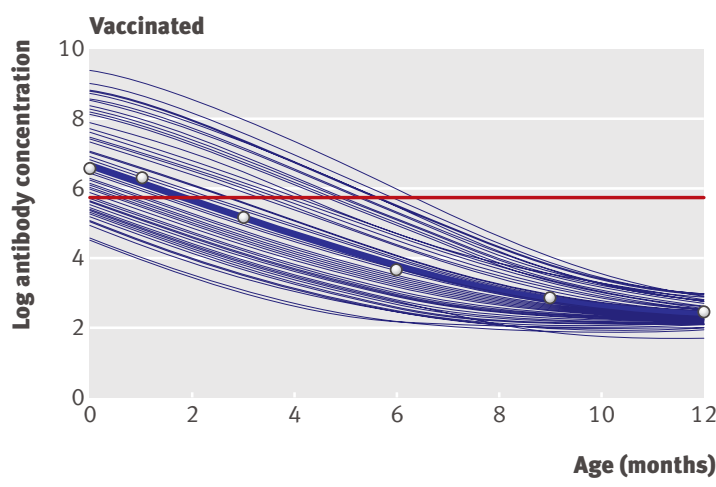

Fig 1| Fitted individual profiles for decay in log antibody concentration $(\log (A L+1))$ based on linear mixed model (blue lines) in all infants, infants from naturally immune women, and infants from vaccinated women. Horizontal red line indicates threshold of $300 \mathrm{mIU} / \mathrm{ml}$. Thick curve is predicted mean curve in each group with observed means at birth and 1 , $3,6,9$, and 12 months as open circles

the vaccinated group and $95 \%$ in the naturally immune group had lost immunity.

Regression of the estimated time to loss of immunity against the $\log$ antibody concentration of the mother gives $\mathrm{R}^{2}=0.97$, indicating a very good predictive value. Figure 3 shows a regression plot with 95\% prediction intervals for the time to loss of immunity for a child, given a certain antibody concentration in the mother. The lower part is the density of log antibody concentration for naturally immune and vaccinated women. For example, if the modus of the log (IgG) of a vaccinated woman was 6.2 , looking at the regression plot, her child will lose maternal antibodies between 0 (lower 95\% prediction limit) and 2 months of age (upper 95\% prediction limit). The child of a naturally immune woman with a modus $\log (\operatorname{IgG})$ of 8 will lose maternal antibodies between 3 and 5 months of age.

\section{DISCUSSION}

The starting concentrations of maternal antibodies in infants in this study depended highly on the concentration of antibodies in the mother and on her vaccination status. Infants of vaccinated women started with significantly fewer antibodies than did infants of naturally immune women. The rate of decay of maternal antibodies was slightly steeper in infants of naturally immune women (fig 2). The median time to loss of immunity was 2.61 months: 0.97 months for infants of vaccinated women and 3.78 months for infants of naturally immune women. We prefer to use the median rather than the mean time to loss of immunity because a quarter of the infants of vaccinated women started at birth with antibody titres below the threshold we used, which influences the mean. We found no significant impact of breast feeding, parity, birth weight, educational level, caesarean section, or day care attendance on the duration of maternal antibodies.

\section{Strengths and weaknesses of study}

The combination of a comprehensive prospective study and up to date statistical methods is innovative. The linear mixed model we used offered the possibility of predicting the time to loss of passive immunity in a child starting from the maternal titre of anti-measles antibody. Taking into account the confidence intervals on the model's prediction, the remaining protected portion at 6 months of age will be $5-15 \%$, which correlates with the serological findings. On the basis of this study, we cannot make a general statement about the prediction of the time to loss of immunity by using the maternal level at 36 weeks for any population. However, we do believe that this study could play a pivotal role in the consideration of individualised vaccination programmes.

A few shortcomings can be recognised. The threshold of the ELISA we used, chosen according to the optical density value, is questionable. However, even if this value is overestimated and values below it are still in the protective range, all antibodies had disappeared by the age of 6 months. Furthermore, the ELISA is commonly used in practice and referred to in the literature. ${ }^{1215-17}$

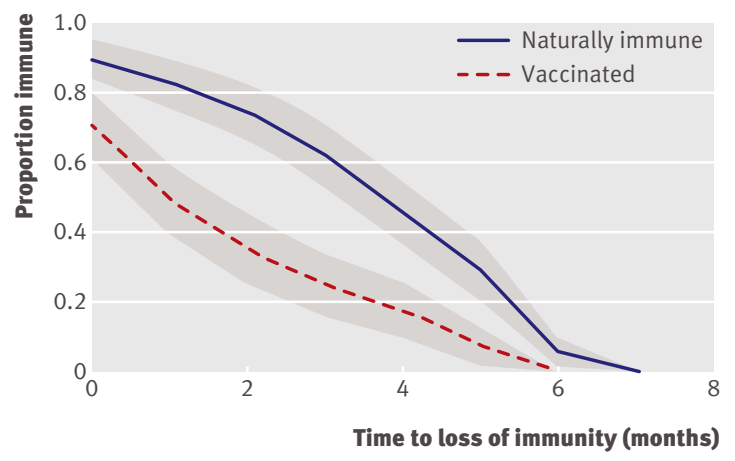

Fig 2 | Proportion of infants of vaccinated women and naturally immune women still immune as a function of time to loss of immunity. Shaded area is $95 \%$ confidence interval 

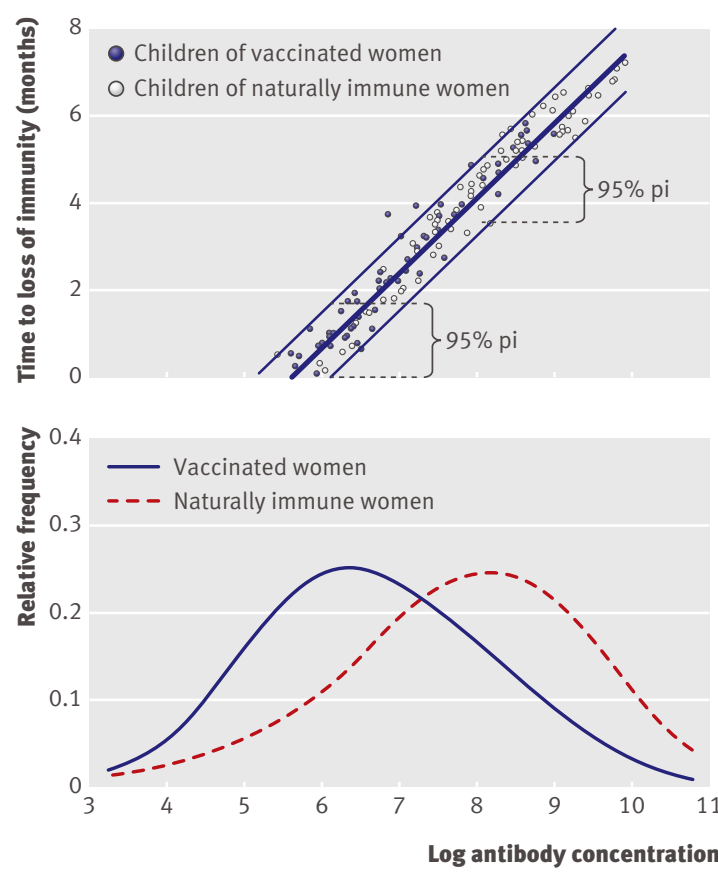

Fig 3 Regression fitted to predicted time to loss of immunity (upper part) with $95 \%$ prediction intervals, and density plots (lower part) of log antibody concentration at week 36 of pregnancy for vaccinated and naturally immune women. $\mathrm{X}$ axis represents log transformation of mother's antibody concentration at week 36

We can question the representative nature of the study population. However, compared with seroprevalence data from 2006 in Flanders, ${ }^{18}$ no significant difference in geometric mean titre against measles exists between the female population at childbearing age in the Province of Antwerp $(1782 \mathrm{mIU} / \mathrm{ml})$ and our study population $(\mathrm{P}=0.1)$.

\section{Comparison with other studies}

Our results confirm data from the literature but support a shortening presence of maternal antibodies. We discussed the relevant publications in a previous review article. ${ }^{8}$ Comparison between publications is difficult owing to the different laboratory tests and different age categories used. In the literature, the mother's year of birth is mostly used as a proxy for her vaccination status. In our study the chance of misclassification is reduced to a minimum. Publications from the 1980s showed that women vaccinated with a live attenuated measles vaccine had lower amounts of antibodies and passed on shorter term protection against measles compared with naturally infected women. ${ }^{1920}$ Pabst did a longitudinal study in the United States (1992) and found that more than $90 \%$ of infants of vaccinated women were susceptible to measles by the age of 7 months compared with $65 \%$ of infants of naturally immune women. ${ }^{21} \mathrm{He}$ found a steeper slope of antibodies in infants of naturally immune women. Brugha et al (UK, 1996) found significantly more infants of vaccinated women between 5 and 7 months of age with low maternal antibody concentrations. ${ }^{22}$ In a study by De Serres et al (Canada, 1997), half of infants of naturally immune women were still protected at 8 months compared with $15 \%$ of infants of vaccinated women. ${ }^{23}$ Klinge et al reported in 2000 that protection in Germany was shortening over time, leaving almost no infants protected at the age of 9 months. ${ }^{24}$ In Switzerland in 2004, 19\% of infants still had positive titres after 6-9 months. ${ }^{25}$ In a recent French study, however, only 10\% of each group were still protected at month $6 .{ }^{26}$ More importantly, no significant difference was found according to the mother's year of birth or reported history of measles.

\section{Policy implications}

The early loss of maternal antibodies in infants has several implications. Recommendations have been made for vaccination at the age of 9 months in epidemic situations, with a second dose at $15-24$ months. ${ }^{2627}$ Early vaccination of infants in case of an outbreak can be defended on the basis of this study, although humoral immune response to measles vaccination has been shown to be less efficient owing to immunological immaturity. ${ }^{24}$ However, T cell responses are sufficiently elicited to prime humoral response to a second dose. ${ }^{28-30}$ If future studies could further show that measles vaccines can be offered at an earlier age (for example, at 9 months or even earlier), policy makers could consider, given our data, moving forward the routine measles vaccination programme. For the moment, vaccination at a young age should be considered in case of a situation of early exposure (such as an outbreak or contact with siblings with measles). Moreover, these results can help with decision making on individual infants travelling or migrating to endemic areas and support ongoing research on early vaccination. Avoiding contact between people with measles and unvaccinated infants is preferable but yet not feasible.

The use of newly developed vaccines - for example, a DNA vaccine that induces immune responses at a very young age - could offer the opportunity of vaccinating very young infants more efficiently. ${ }^{8}$ Additionally, these vaccines would have a beneficial effect on immunological memory. ${ }^{3}$ Our data underline the need for further research on early administration of vaccine.

Reduction in incidence and epidemics of measles could be achieved with timely vaccination. ${ }^{3132}$ Several studies have recently shown that important percentages of children are immunised with delay. ${ }^{10113334} \mathrm{~A}$ US study reported that $11 \%$ of children had measles vaccination delayed for six to eight months during the first 24 months of life. ${ }^{33}$ The risk of disease due to delay depends on several factors, such as circulation of disease, transmissibility, likelihood of importation, and severity of outcome. ${ }^{31}$ Our data can only strengthen the need for timely administration of a first dose of measles containing vaccine.

Giving booster doses to women at childbearing age could be a solution to the low amount of maternal antibodies. However, a second vaccine dose consolidates immunity but does not augment the titre of antibodies over the long term. As a consequence, the amount of 


\section{WHAT IS ALREADY KNOWN ON THIS TOPIC}

Infants of women vaccinated against measles receive fewer maternal antibodies and thus have shorter protection than infants of women with naturally acquired immunity

Whether the amount of maternal antibodies is still sufficient to protect infants until the first dose of vaccine is administered in the era of changing measles epidemiology is not known

\section{WHAT THIS STUDY ADDS}

An increasing gap of susceptibility exists between the loss of maternal antibodies and the administration of a first dose of vaccine

This is true not only in infants of vaccinated women but also in infants of women with naturally acquired immunity

This study underlines the importance of timeliness of administration of a first measles containing vaccine at 12 months of age

maternal antibodies transferred to infants will not be influenced substantially. ${ }^{35-37}$ Vaccination of pregnant women is a last option. This ethically burdened discussion goes beyond the scope of this paper.

\section{Conclusions}

Decision makers and clinicians should be aware of the early loss of maternal protection, and each country should monitor its own situation. Clinical recognition of cases of measles in young infants is important for surveillance of the disease. These results can help with decision making on individual infants travelling or migrating to endemic areas and support ongoing research on early vaccination. Most importantly, we confirm the extreme importance of timely administration of the first dose of measles vaccine.

Contributors: EL and PVD were responsible for the conception and design of the study. VH, EL, and MI did the laboratory tests. EL, NH, and MA analysed and interpreted the data. EL, NH, and PVD drafted the manuscript and completed critical revisions. All authors approved the final manuscript. EL is the guarantor.

Funding: EL obtained a research grant from the Faculty of Medicine at the University of Antwerp. An unrestricted educational grant from GlaxoSmithKline Belgium, Genval, covered part of the nursing activities. The study sponsors had no role in the study design; the collection, analysis and interpretation of the data; the writing of the report; or the decision to submit the article for publication.

Competing interests: None declared.

Ethical approval: Study protocol and documents were reviewed and approved by the ethics committee at each participating institution; the leading ethics committee is based at the University Hospital in Antwerp. We thank all the participating women and their children. Data sharing: No additional data available.

1 World Health Organization. Progress in global measles control and mortality reduction 2000-2007. Wkly Epidemiol Rec 2008;83:441-8,

2 Muscat M, Bang H, Wohlfahrt J, Glismann S, Molbak K. Measles in Europe: an epidemiological assessment. Lancet 2009;373:383-9.

3 Zinkernagel RM. Advances in immunology: maternal antibodies, childhood infections, and autoimmune diseases. $N$ Engl/ Med 2001;345:1331-5.

4 Gans H, DeHovitz R, Forghani B, Beeler J, Maldonado Y, Arvin AM. Measles and mumps vaccination as a model to investigate the developing immune system: passive and active immunity during the first year of life. Vaccine 2003;21:3398-405.

5 Glezen WP. Effect of maternal antibodies on the infant immune response 3. Vaccine 2003;21:3389-92.

6 Dagan R, Slater PE, Duvdevani P, Golubev N, Mendelson E. Decay of maternally derived measles antibody in a highly vaccinated population in southern Israel. Pediatr Infect Dis / 1995;14:965-9.

7 Linder N, Tallen-Gozani E, German B, Duvdevani P, Ferber A, Sirota L. Placental transfer of measles antibodies: effect of gestational age and maternal vaccination status. Vaccine 2004:22:1509-14.
8 Leuridan E, Van Damme P. Passive transmission and persistence of naturally acquired or vaccine-induced maternal antibodies against measles in newborns. Vaccine 2007:25:6296-304.

9 Caceres VM, Strebel PM, Sutter RW. Factors determining prevalence of maternal antibody to measles virus throughout infancy: a review. Clin Infect Dis 2000;31:110-9.

10 Akmatov MK, Kretzschmar M, Kramer A, Mikolajczyk RT. Timeliness of vaccination and its effects on fraction of vaccinated population. Vaccine 2008;26:3805-11.

11 Luman ET, Chu SY. When and why children fall behind with vaccinations: missed visits and missed opportunities at milestone ages. Am J Prev Med 2009;36:105-11.

12 Vandermeulen C, Mathieu R, Geert LR, Pierre VD, Karel H. Long-term persistence of antibodies after one or two doses of MMR-vaccine. Vaccine 2007;25:6672-6.

13 Beutels P, Van Damme P, Van Casteren V, Gay NJ, De Schrijver K, Meheus A. The difficult quest for data on "vanishing" vaccinepreventable infections in Europe: the case of measles in Flanders (Belgium). Vaccine 2002;20:3551-9.

14 Verbeke M. Linear mixed models for longitudinal data. Springer Verlag, 2000.

15 Scott S, Cumberland P, Shulman CE, Cousens S, Cohen B), Brown DW, et al. Neonatal measles immunity in rural Kenya: the influence of HIV and placental malaria infections on placental transfer of antibodies and levels of antibody in maternal and cord serum samples. J Infect Dis 2005;191:1854-60.

16 Kremer IR, Schneider F, Muller CP. Waning antibodies in measles an rubella vaccines-a longitudinal study. Vaccine 2006;24:2594-601.

17 Tischer A, Andrews N, Kafatos G, Nardone A, Berbers G, Davidkin I, et al. Standardization of measles, mumps and rubella assays to enable comparisons of seroprevalence data across 21 European countries and Australia. Epidemiol Infect 2007;135:787-97.

18 Theeten HHV, Dedeakayogullari H, Baetens D, Billiet J, Couck P, Dediste A, et al. Seroprevalence of vaccine preventable diseases in Belgium. Pediatr Infect Dis / 2009;28:e75.

19 Jenks P. Maternally derived measles immunity in children of naturally infected and vaccinated mothers. Epidemiol Infect 1988;101:473-6.

20 Lennon IL, Black FL. Maternally derived measles immunity in era of vaccine-protected mothers. J Pediatr 1986;108:671-5.

21 Pabst HF, Spady DW, Marusyk RG, Carson MM, Chui LWL, Joffres MR, et al. Reduced measles immunity in infants in a well-vaccinated population. Pediatr Infect Dis / 1992;11:525-9.

22 Brugha R, Ramsay M, Forsey T, Brown D. A study of maternally derived measles antibody in infants born to naturally infected and vaccinated women. Epidemiol Infect 1996;117:519-24.

23 De Serres G, Joly JR, Fauvel M, Meyer F, Masse B, Boulianne N. Passive immunity against measles during the first 8 months of life of infants born to vaccinated mothers or to mothers who sustained measles. Vaccine 1997;15:620-3.

24 Klinge J, Lugauer S, Korn K, Heininger U, Stehr K. Comparison of immunogenicity and reactogenicity of a measles, mumps and rubella (MMR) vaccine in German children vaccinated at 9-11,12-14 or 15-17 months of age. Vaccine 2000;18:3134-40.

25 Leineweber B, Grote V, Schaad UB, Heininger U. Transplacentally acquired immunoglobulin $\mathrm{G}$ antibodies against measles, mumps, rubella and varicella-zoster virus in preterm and full term newborns. Pediatr Infect Dis / 2004;23:361-3.

26 Gagneur A, Pinquier D, Aubert M, Balu L, Brissaud O, De Pontual L, et al. Kinetics of decline of maternal measles virus-neutralizing antibodies in sera of infants in France in 2006. Clin Vaccine Immunol 2008;15:1845-50.

27 Gans HA, Lew-Yasukawa L, Beeler J, DeHovitz R, Maldonado Y, Arvin AM. Immune responses of 6,9 and 12 month old infants immunized with measles or mumps vaccine and the effects of passive antibodies on these responses. Pediatr Res 1999;45:161A

28 Gans HA, Yasukawa LL, Alderson A, Rinki M, DeHovitz R, Beeler I et al. Humoral and cell-mediated immune responses to an early 2dose measles vaccination regimen in the United States. J Infect Dis 2004;190:83-90.

29 Siegrist CA. Mechanisms by which maternal antibodies influence infant vaccine responses: review of hypotheses and definition of main determinants. Vaccine 2003;21:3406-12.

30 Siegrist CA. Vaccination in the neonatal period and early infancy. Intern Rev Immunol 2000;19:195-219.

31 Dannetun E, Tegnell A, Hermansson G, Torner A, Giesecke J. Timeliness of MMR vaccination-influence on vaccination coverage. Vaccine 2004;22:4228-32.

32 Heininger $U$, Zuberbuhler $M$. Immunization rates and timely administration in pre-school and school-aged children. Eur J Pediatr 2006;165:124-9.

33 Luman ET, Barker LE, McCauley MM, Drews-Botsch C. Timeliness of childhood immunizations: a state-specific analysis. Am J Public Health 2005;95:1367-74 
34 Clark A, Sanderson C. Timing of children's vaccinations in 45 lowincome and middle-income countries: an analysis of survey data. Lancet 2009;373:1543-9.

35 Chen RT, Markowitz LE, Albrecht P, Stewart JA, Mofenson LM, Preblud SR, et al. Measles antibody-reevaluation of protective titers. J Infect Dis 1990;162:1036-42.

36 Deseda-Tous J, Cherry JD, Spencer MJ, Welliver RC, Boyer KM, Dudley JP, et al. Measles revaccination: persistence and degree of antibody titer by type of immune response. Am J Dis Child 1978;132:287-90.

37 Pebody RG, Gay NJ, Hesketh LM, Vyse A, Morgan-Capner P, Brown DW, et al. Immunogenicity of second dose measles-mumps rubella (MMR) vaccine and implications for serosurveillance. Vaccine 2002;20:1134-40.

Accepted: 25 January 2010 